https://doi.org/10.46813/2021-133-038

\title{
PROJECT OF A BEAMLINE FOR THE NESTOR STORAGE RING LINAC TO PROVIDE NUCLEAR PHYSICS RESEARCH
}

\author{
V.V. Mytrochenko, S.O. Perezhogin, V.A. Kushnir, V.B. Ganenko, M.I. Ayzatsky, \\ G.D. Kovalenko \\ National Science Center "Kharkov Institute of Physics and Technology”, Kharkiv, Ukraine \\ E-mail: mitvic@kipt.kharkov.ua
}

The results of self-consistent beam dynamics simulation are presented for the linac of the NESTOR storage ring. Consideration is given to the influence of particle energy change at the output of the electron gun during the current pulse. The obtained data have formed the basis for the computation of the beam transport line for research in the field of high energy physics.

PACS: 41.75.Ht, 41.85.Ja

\section{INTRODUCTION}

The NESTOR storage ring, designed to generate soft X-rays [1], is under commissioning at NSC KIPT. It was intended to use the linac [2] both as an injector into the ring and for research in nuclear physics at a straight section of a beamline. However, this straight section has not yet been established. The aim of this work was more extensive study by numerical simulation of selfconsistent particle dynamics in the linac to refine the beam characteristics at its output and to develop the straight section of the beamline for transporting this beam to a goniometer installed at the entrance of a "dog-leg" beamline section of the former LU-300 linac.

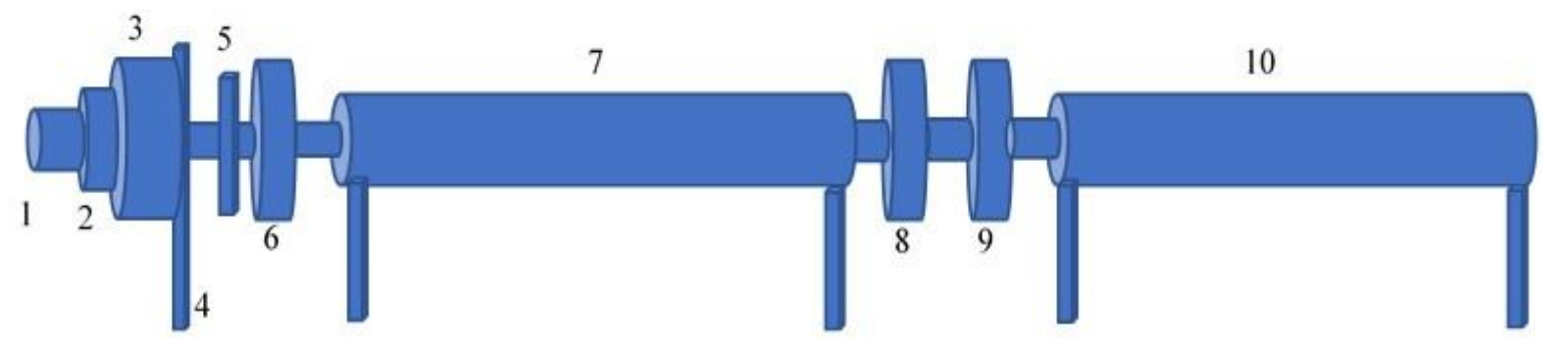

Fig. 1. Schematic representation of the accelerator-injector of the NESTOR ring

The linac operation has some features. First, it is not possible to vary the delay of the microwave power pulses feeding the accelerating sections relative to each other to compensate the influence of transients on output beam parameters because of the RF feeding scheme peculiarities. In addition, the high-voltage power supply of the electron gun has approximately $10 \%$ pulse overshoot. Therefore, to determine the output beam parameters, it is necessary to simulate the self-consistent dynamics of particles in the linac in the time domain. Previously, we developed a technique for such simulation [6], but it did not consider the change in energy of the initial beam within a current pulse, which was generated by the electron gun. Thus, it was necessary to develop an addition to the method [6], to consider that change and simulate the self-consistent particle dynamics in the linac, schematically shown in Fig. 1 in the time domain, taking into account the change in initial beam energy.

\section{FORMULATION OF THE PROBLEM}

The linac-injector of the NESTOR ring is shown schematically in Fig. 1. It consists of a diode electron gun 1, which accelerates a beam with a current of $0.2 \mathrm{~A}$ to an energy of $25 \mathrm{keV}$, an injector on evanescent wave 2 [3] with a coaxial coupler 4, which accelerates particles to an energy of $1 \mathrm{MeV}$ and two traveling wave accelerating sections 7 and 10, the first of which is the section "Kharkiv-85" [4], and the second is the LU-60 section [5]. These sections provide particle energy at the exit of the accelerator up to $100 \mathrm{MeV}$. The beam transport consists of two axially symmetric magnetic lenses 3 and 6 and two quadrupole lenses 8 and 9 . A collimator 5 is used to regulate the beam current at the accelerator exit.

\section{SIMULATION OF SELF-CONSISTENT PARTICLE DYNAMICS}

To simulate the self-consistent particle dynamics with the developed method [6], which uses, in particular, modeling of particle dynamics within the framework of the PARMELA code [7], one needs to know a list of electrodynamic characteristics of resonant systems, including on-axis field distribution, magnetic characteristics of axially symmetric magnetic lenses, and such parameters of the accelerating sections as group velocity, attenuation and series impedance. These data for axially symmetric resonant and magnetic systems were calculated using the SUPERFISF/POISON group of codes [8]. The parameters of the accelerating sections were calculated using the method [9].

\subsection{PARAMETERS OF THE ACCELERATING SYSTEM}

The simulated characteristics of the injector resonant system, which consists of five coupled cavities that are excited in the stopband, are given in Table 1. 
Table 1 particles during a current pulse. We used input 60 to

Electrodynamic characteristics of the injector

\begin{tabular}{|l|l|}
\hline Parameter & Value \\
\hline Operating frequency $\mathrm{f}_{0}, \mathrm{MHz}$ & 2797.15 \\
\hline Quality factor Q & 12354 \\
\hline Shunt impedance, $\mathrm{M} \Omega / \mathrm{m}$ & 18.4 \\
\hline Power losses in the walls, $\mathrm{kW}$ & 558 \\
\hline Maximum field on the axis, MW/m & 39.4 \\
\hline $\begin{array}{l}\text { The coupling coefficient of the } \\
\text { waveguide with the resonant system } \beta\end{array}$ & 3.8 \\
\hline
\end{tabular}

Accelerating sections are cylindrical waveguides loaded with disks, consisting of several subsections with constant impedance. Each homogeneous part is combined with the next one by means of five transient cells. The total number of cells in the "Kharkiv-85" section is 162 , which are divided into four homogeneous subsections, and the LU-60 section has 102 cells, which are divided into three homogeneous subsections. The wave phase shift per a cell is $90^{\circ}$, at the operating frequency of $2797.15 \mathrm{MHz}$, which corresponds to the structure period of $2.679 \mathrm{~cm}$. The disks thickness is $0.4 \mathrm{~cm}$. Therefore, the "Kharkiv-85" section has 19 disks with different aperture, and the LU-60 section, respectively, has 13 ones. According to the method [9], the 19 and 13 representative resonant stacks were modeled, respectively. The eigen mode that corresponded to the traveling wave with a phase shift per period of $90^{\circ}$ was considered. Using the field distribution on the stack axis, $Q-$ factor and stored energy, the following parameters were calculated: relative group velocity $\beta_{g}$; attenuation $\alpha$, series impedance $R_{s}$; shunt impedance $Z$. When modeling a spatially inhomogeneous section using the method of [6], several assumptions are made, in particular, about the locality of the inhomogeneity with a slow change in the parameters along the section. It is assumed that the parameters of the section on the period, which includes a disk with a certain size of the aperture, is determined exclusively by this aperture. Using the same approach, the generalized characteristics of the sections were calculated, which are given in Table 2.

\subsection{INJECTION ENERGY CHANGE}

In the PARMELA program, initial parameters of particles, including their energy, are specified in several ways, depending on the selected type of generation of initial array, which is specified by the keyword input in the input file of the program. For input 0 , the parameters of each particle are written to the input file diout. For input 3, the particles are generated according to the data calculated using the EGUN program. Therefore, these methods are not convenient for changing the energy of generate particles. In this case, the parameters of the particles in the transverse plane are determined randomly, to fill the given ellipses in the planes $\mathrm{x}-\mathrm{x}^{\prime}$ and $\mathrm{y}-$ $y^{\prime}$, and in the longitudinal direction - evenly fill the given intervals of phases and energies that are tied to reference particle. The initial phase (actually, its position) and the energy of the reference particle are given by the keyword run. If the electron gun operates in the mode of current limitation by the space charge, the gun current is changed with a gun voltage in accordance with the Child-Langmuir law (law " $3 / 2$ ") while the transverse characteristics of the beam remain unchanged. Thus, finding the characteristics of the gun beam at a known voltage using the EGUN program, we can easily simulate the dynamics of the beam at any voltage, changing only the initial position and energy of the reference particle and the beam current.

Table 2

Characteristics of accelerating sections

\begin{tabular}{|l|l|l|}
\hline \multicolumn{1}{|c|}{ Parameter } & $\begin{array}{c}\text { "Kharkiv- } \\
85 "\end{array}$ & LU-60 \\
\hline Operating frequency $\mathrm{f}_{0}, \mathrm{MHz}$ & 2797.15 & 2797.15 \\
\hline $\begin{array}{l}\text { Phase shift per a period of the } \\
\text { structure, deg. }\end{array}$ & 90 & 90 \\
\hline Length, $\mathrm{m}$ & 4.31 & 3.22 \\
\hline $\begin{array}{l}\text { The number of homogeneous } \\
\text { subsections }\end{array}$ & 4 & 3 \\
\hline $\begin{array}{l}\text { The maximum radius of the } \\
\text { aperture } a, \mathrm{~mm}\end{array}$ & 12.72 & 10.91 \\
\hline $\begin{array}{l}\text { The minimum radius of the } \\
\text { aperture } a, \mathrm{~mm}\end{array}$ & 9.81 & 8.3 \\
\hline Filling time, $\mu \mathrm{s}$ & 0.915 & 1.22 \\
\hline Attenuation, Neper & 0.68 & 0.914 \\
\hline $\begin{array}{l}\text { Average series impedance, } \\
\Omega / \mathrm{cm}\end{array}$ & 1814 & 3495 \\
\hline Beam loading, MeV/A & 65.6 & 64.5 \\
\hline $\begin{array}{l}\text { Energy gain at a microwave } \\
\text { power of 16 MW and zero } \\
\text { current, MeV }\end{array}$ & 54 & 51 \\
\hline
\end{tabular}

The gun voltage pulse is characterized by the following parameters: $t_{0}$ is delay of pulse start, $\tau_{\text {rise }}$ is the pulse risetime, $\tau_{\text {flat }}$ is duration of the pulse flat-top, $\tau_{\text {fall }}$ is pulse fall time, $V_{s t}$ is amplitude of the steady part of the pulse and $M$ is the pulse overshoot. The total pulse duration is equal to $\tau_{\text {rise }}+\tau_{\text {flat }}+\tau_{\text {fall }}$. The voltage $V(t)$ on the gun before the beginning of the pulse and after the pulse end is zero while during the pulse it is determined by the formula:

$$
V(t)=\left\{\begin{array}{c}
\frac{V_{\text {st }} \cdot M}{2}\left(1-\cos \left(\frac{\pi\left(t-t_{0}\right)}{\tau_{\text {rise }}}\right)\right), t_{0}<t<t_{0}+\tau_{\text {front }}, \\
V_{\text {st }} \cdot\left(e^{-\left(\frac{t-\left(t_{0}+\tau_{\text {rise }}\right)}{2 \tau_{\text {rise }}}\right)^{2}}(M-1)+1\right), t_{0}+\tau_{\text {rise }}<t<t_{0}+\tau_{\text {rise }}+\tau_{\text {flat }}, \\
\frac{V_{\text {st }}}{2} \cdot\left(1-\cos \left(\frac{\pi\left(t-t_{0}-\tau_{\text {front }}-\tau_{\text {flat }}\right)}{\tau_{\text {fall }}}+\pi\right)\right), t_{0}+\tau_{\text {rise }}+\tau_{\text {flat }}<t<t_{0}+\tau_{\text {front }}+\tau_{\text {flat }}+\tau_{\text {fall }} .
\end{array}\right.
$$

The energy of the reference particle at each moment of simulation is determined from (1) and is written to beam current is determined by the formula: 


$$
I(t)=\mu V(t)^{\frac{3}{2}}
$$

where $\mu$ is gun perveance.

During the simulation, it was found that at low initial energy of the beam, the particles do not reach the end of the section, which led to a violation of the simulation algorithm. It was found that simulation is only possible for the gun voltage more than $20500 \mathrm{~V}$. Therefore, not the entire voltage pulse on the gun was simulated, but only that part where the voltage exceeded $20500 \mathrm{~V}$. In addition, for stable and faster operation of the simulation algorithm, an application was developed that allows one to read the binary output file of the PARMELA code with the parameters of accelerated particles. This greatly speeds up the algorithm and allows one to simulate more particles, compared to reading a text file, as was the case in the previous version.

The shape of the RF power pulse was given by a formula similar to (1), with the difference that the peak of the pulse is flat with amplitude $P_{s t}$, without overshoot. During the pulse, only the RF power changed, but the phase $\Phi_{s t}$ remained constant for the whole pulse.

If a simulated system has two or more microwave power sources, such as an injector and a first section the time parameters of the RF power pulses will be the same, but the amplitudes and phases may be different.

\subsection{SIMULATION RESULTS}

During the simulations we investigated some dependencies that have operational significance. They are following: the dependence of the output current at the first section exit on the injector solenoid field; the dependence of the output beam parameters of the first section on the phase of its RF power supply; the dependence of the output beam parameters of the linac on RF power supply phases of the first and the second sections. Taking into account the age of the klystron amplifiers used to power the accelerator sections, we used rather moderate values of the RF power of the power supply sections equal to $12 \mathrm{MW}$, which corresponds to the experimental data.

The simulation parameters of the first section with the injector were as follows. Voltage on the gun and perveance were $24000 \mathrm{~V}$ and $6.3 \cdot 10^{-8}$, respectively. The pulse parameters of the gun $\tau_{0}, \tau_{\text {rise }}, \tau_{\text {flat }}, \tau_{\text {fall }}$ and $M$ were $1.07,0.50,1.29,0.50 \mu$ s and 1.1 , respectively. The time parameters of the microwave pulse $\tau_{\text {flat }}, \tau_{\text {rise }}$, $\tau_{\text {fall }}$ were $2.50,0.50,0.50 \mu \mathrm{s}$, respectively. The parameters of the microwave power of the injector $P_{s t}, \Phi_{s t}$, $\beta$ were $1 \mathrm{MW}, 45$ degree and 5 , respectively. The parameters of the microwave power supply of the first section $P_{s t}$ and $\Phi_{s t}$, were $12 \mathrm{MW}$ and 45 degree through 115 degree, respectively. The microwave phase of the first section from 45 to 115 degrees corresponds to the achievement of the minimum phase length of the bunches and the maximum energy gain of particles, respectively. Results obtained are listed in Table 3.

In Table 3: $I_{b}$ is the current of the output beam, $W_{a v}$ is the average energy of the beam particles, $W_{p i k}$ is energy of the particles in the energy spectrum peak, $\Delta W / W_{(70 \%)}$ is the relative width of the energy range that includes $70 \%$ of the beam particles, $\Delta W / W_{F W H M}$ is the 40 relative width of the energy spectrum at half the distribution height, $\Delta \Phi_{(70 \%)}$ is the width of the phase spectrum, which includes $70 \%$ of the beam particles, $\Delta \mathrm{x}_{(70 \%)}$ is the beam size in the transverse coordinate $\mathrm{x}$, which includes $70 \%$ of the beam particles, $\Delta y_{(70 \%)}$ is a similar value for the coordinate y, $\Delta x_{r m s}$ and $\Delta y_{r m s}$ are the root mean square (rms) dimensions of the beam in the transverse direction $\mathrm{x}$ and $\mathrm{y}$, respectively, $\varepsilon_{x n \text { rms }}$ and $\varepsilon_{y n r m s}$ are the normalized rms emittances in the transverse planes, $\Phi_{r f} 1$ sect. or $\Phi_{r f} 2$ sect. phases of the first or the second section RF power supply. Solenoid multiplier is the coefficient by which the solenoid field calculated by the POISSON program is multiplied at the PARMELA code simulation. Carried out simulation of beam parameters at the exit of the first section on the solenoid multiplier has shown that the output current and the beam size are weakly dependent on the solenoid field. Therefore, at further simulation the multiplier of the solenoid field was set to 1 .

Table 3

Dependence of beam parameters on microwave phase of the first section

\begin{tabular}{|l|l|l|l|l|l|}
\hline$\Phi_{r f 1 \text { sect }}$, deg. & 45 & 55 & 75 & 95 & 115 \\
\hline$I_{b}, \mathrm{~mA}$ & 52 & 70 & 97 & 99 & 99 \\
\hline$W_{a v}, \mathrm{MeV}$ & 36 & 35 & 36 & 40 & 42 \\
\hline$W_{p i k}, \mathrm{MeV}$ & 35 & 34 & 37 & 40 & 41 \\
\hline$\Delta W / W_{(70 \%)}, \%$ & 4.6 & 7 & 8.7 & 6.8 & 4.3 \\
\hline$\Delta W / W_{F W H M}, \%$ & 1 & 3.9 & 8.7 & 6.3 & 0.5 \\
\hline$\Delta \Phi_{(70 \%)}, \mathrm{deg}$. & 1.4 & 2.8 & 5.6 & 8.9 & 11 \\
\hline$\Delta x_{, y_{(70 \%)}, \mathrm{cm}}$ & 0.98 & 0.95 & 0.69 & 0.35 & 0.17 \\
\hline$\Delta x, y_{r m s}, \mathrm{~cm}$ & 0.43 & 0.42 & 0.34 & 0.19 & 0.09 \\
\hline$\varepsilon_{x, y n \text { rms }}, \mathrm{mm} \cdot \mathrm{mrad}$ & 43 & 46 & 29 & 11 & 6 \\
\hline $\begin{array}{l}\text { Solenoid multi- } \\
\text { plier }\end{array}$ & 1 & 1 & 1 & 1 & 1 \\
\hline
\end{tabular}

Let us analyze data in Table 3 . It can be seen that the energy of the beam increases almost monotonically with increasing phase shift of the section, while the width of the energy spread behaves differently. At the maximum energy gain, the spread width is the smallest, but at the minimum phase width of the bunches, it is also quite small, having a maximum within the interval. It is known that the minimum energy spread in the resonant accelerator is observed when accelerating bunches at the crest of an electromagnetic wave. It was shown, for example, in $[10,11]$, there are two cases when accelerated bunches became at the wave crest at the exit of the section. The first one is when bunches injected into the section near the crest of accelerating wave. In this case particles of bunch core obtain maximum energy gain, but phase motion of particles is small, so bunch phase length is almost unchanged during acceleration. In another case bunches are injected almost $90^{\circ}$ ahead of the wave crest and particles of bunch core obtain phase dependent longitudinal kicks. Because wave velocity is higher than particle velocities bunches slip to the wave crest at some distance, then phase motion of particles almost stop, but during this slippage bunches undergo strong phase compression. Such behavior is so-called velocity bunching of particles. 
It seems from Table 3 that the phase length of the bunches in the mode of velocity bunching is an order of magnitude smaller than in the mode of maximum energy gain. However, the mode of velocity bunching leads to loss of particles (beam current is almost twice less). At the velocity bunching particles obtain phasedependent increments of both longitudinal and transverse momentum that increases transverse beam emittance and, therefore, leads to beam particle losses. Comparison of images of particles in the transverse phase plane at the output of the first section at the maximum energy gain mode and the mode of minimum phase length of the bunches are shown in Fig. 2. It can be seen that in the last case the particles occupy the entire aperture of the diaphragms (see Table 2), and the shape of the transverse emittance has the form of a socalled "open fan" caused by phase-dependent increments of transverse momentums.

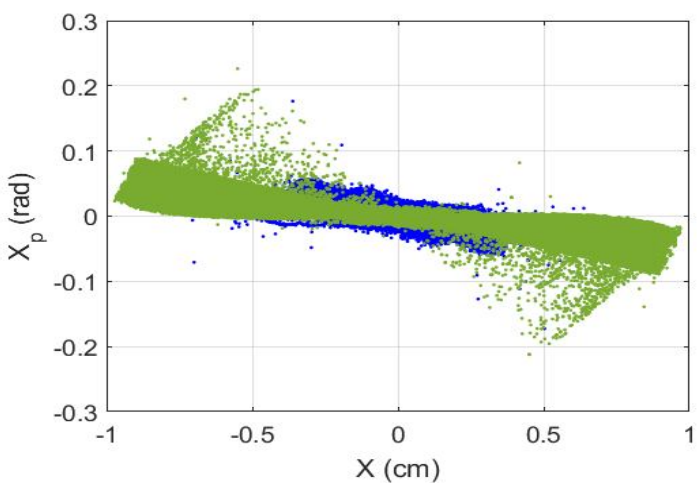

Fig. 2. Image of particles on the transverse phase plane at the exit of the first section at the maximum set of energy (blue dots) and the minimum phase length of the clots (green dots)

It should also be noted that a significant reduction in the accelerated current in the velocity bunching mode compensates the reduction in the energy gain of the particles as these accelerating sections have a significant beam loading of approximately $6.5 \mathrm{MeV}$ per $100 \mathrm{~mA}$ of current (see Table 2).

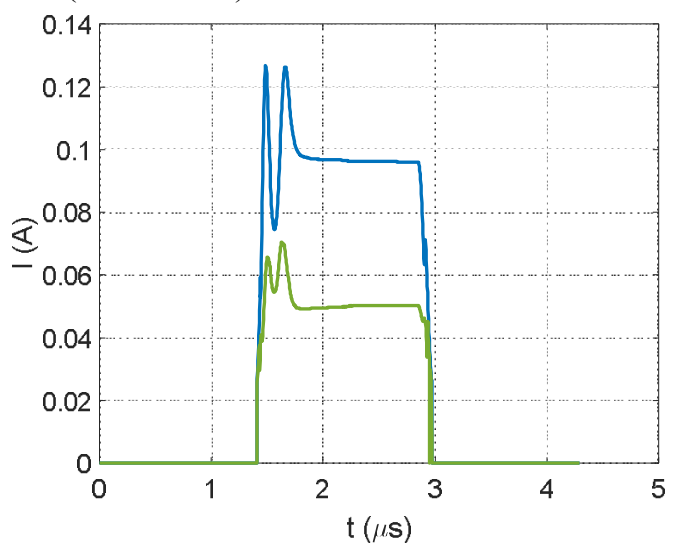

Fig. 3. Pulse current pulses at the output of the first section at the maximum energy set (blue curve) and the minimum phase length of the clots (green curve)

Comparison of current pulses for the cases of the largest energy gain and the minimal phase length of bunches is shown in Fig. 3. One can see that the pulses differ not only in amplitude but also in shape. It should be noted that simulated pulses are similar to the experimentally measured ones.
Comparison of the beam energy spectra at the output of the first section in the maximum energy gain mode and the mode of minimum phase length of the bunches, is shown in Fig. 4. As it can be seen, the maximum energy of the particles in both cases is the same, but the shape of the spectra is quite different. In the case of the minimum phase length of the bunches, a small second peak in the high-energy region is clearly visible on the spectrum. It corresponds to the particles that receive the maximum increase in energy in the steady state. In this case the main part (core) of the bunch has an energy lower than the maximum value due to the specific motion of the particles in the longitudinal phase space as it was mentioned above. This movement leads to the formation of short bunches, as seen in Fig. 5, on which the phase spectra of the beam for the two modes are presented.

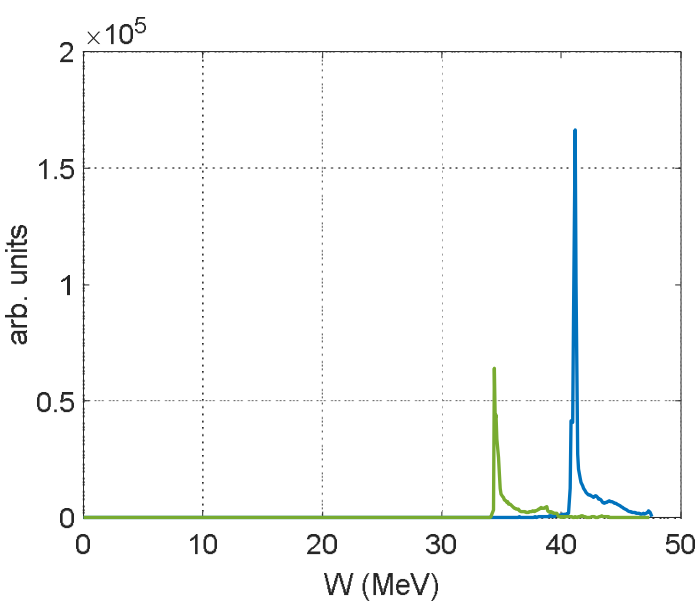

Fig. 4. Energy spectra of the beam at the output of the first section at the maximum energy gain (blue curve) and the minimum phase length of the bunches (green curve)

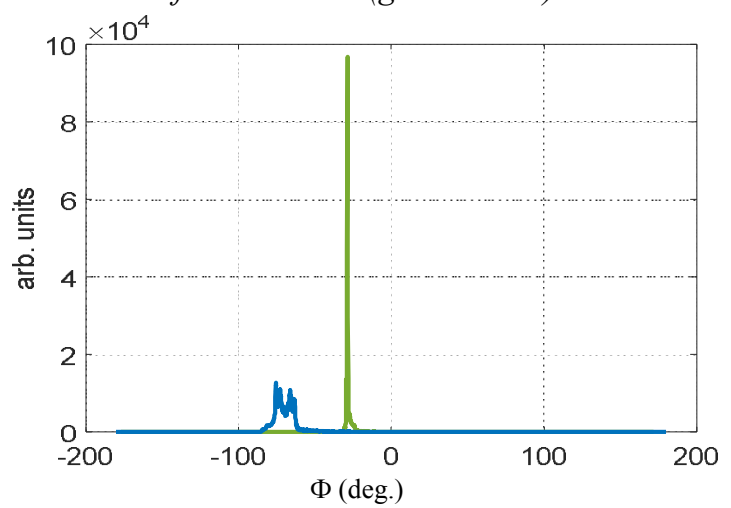

Fig. 5. Phase spectra of the beam at the output of the first section at the maximum energy gain (blue curve) and the minimum phase length of the bunches (green curve)

From Fig. 6 we can get an additional explanation of the behavior of the energy spectra shown in Fig. 5 in the region of energies above the main peak. The presence of particles there is also due to the influence of the transient process caused by the beam loading of the section. It is seen that the average energy of the beam is only set on the second half of the pulse, while in the first half it changes significantly. It should be noted that in the mode of maximum energy gain, the energy spread width at initial part of the current pulse is much smaller than 
that in the case of the minimum phase length of the bunches. This explains the fact that the maximum energy of the particles in both cases is the same.

There are many combinations of microwave power phases of the two sections of the accelerator. However, below we present only the results for the two main modes of microwave power supply of the first section when changing the phase of the microwave power supply of the second section in the range close to 100 degrees at a power of its microwave power supply of $12 \mathrm{MW}$. Quadrupole lenses mounted between the sections were used to guide the beam through the second section. Lens modes were set to obtain approximately the same beam size in the vertical and horizontal planes.

The dependence of the beam parameters at the output of the second section for the case of the minimum length of bunches on the microwave phase of the power supply of this section within 90 degrees was studied. The results showed that the phase change has almost no effect on the beam current and its transverse emittance in this case. The phase length of the bunches and the width of the energy spread of the particles do not change much, while the energy of the beam varies by about $30 \mathrm{MeV}$. Due to the change in the energy of the beam, its dimensions and shape in the transverse plane change somewhat.
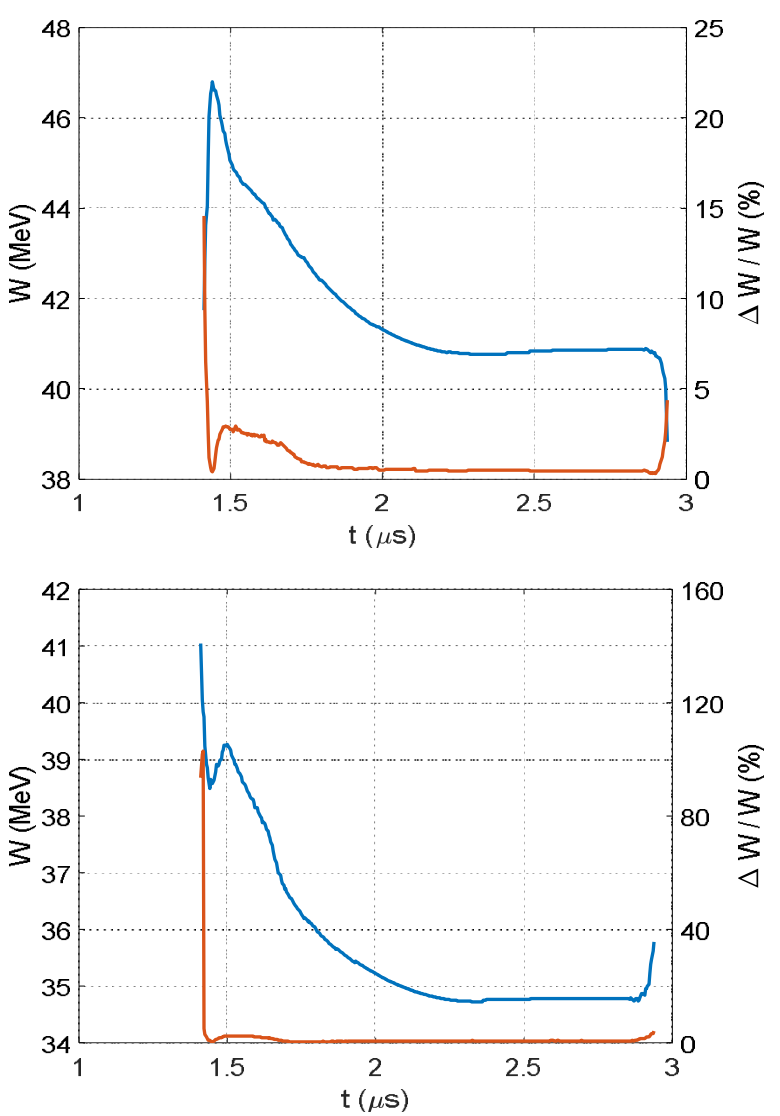

Fig. 6. The average energy of the beam (blue curve) and the width of the energy spectrum (red curve) during the pulse at the output of the first section at the maximum energy gain (top graph) and at the minimum phase length of the bunches (lower graph)

For the case of the maximum energy gain in the first section, the dependence of the beam parameter at the output of the second section on the microwave phase of the power supply of this section was investigated within 80 degrees. Studies have shown that the beam current and its transverse emittance also practically do not change. A smaller change in the energy of the beam occurs in this case, and a change in the width of the energy spread, on the contrary, is greater. Also, a smaller change in the beam dimensions is observed, which is due to a smaller range of changes in the beam energy.

The dependence of the beam energy and the width of the energy spectrum on the phase of the RF power supply of the second section for the two modes of operation of the first section are presented in Fig. 7. The figure shows that for the first mode (the mode of the minimum phase length of bunches), the change in the width of the energy spread of particles is almost an order of magnitude smaller than that for the second mode (maximum energy gain in the first section). In addition, when changing the phase of the microwave power supply of the second section by 20 degrees (from 140 to 160 degrees), the energy of the particles does not change for the first mode.
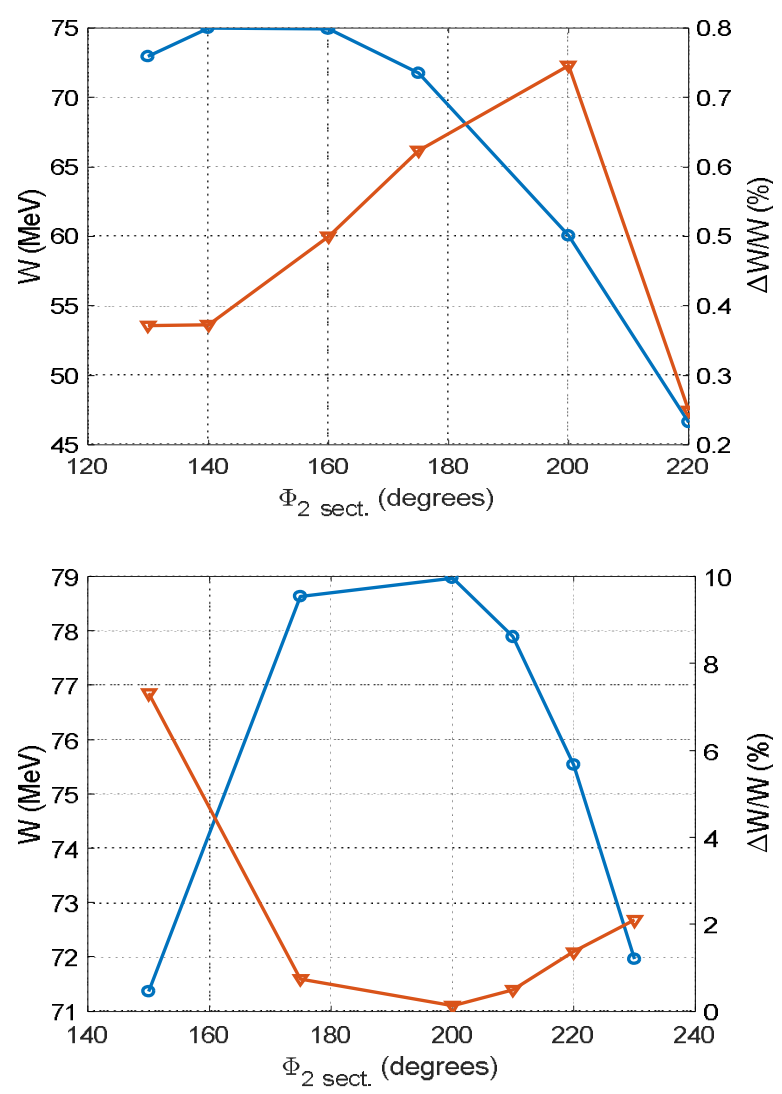

Fig. 7. From above - the dependence of the beam energy (circles) and the beam energy spread (triangles) at the output of the second section on the phase of its microwave power at the minimum phase length of the bunches at the output of the first section. Below are the same at the maximum energy gain at the output of the first section

A comparison of the energy spectra of the beam at the output of the second section for the cases that corresponds to the maximum energy of the beam at the output of the accelerator for two modes of operation of the first section is shown in Fig. 8. 


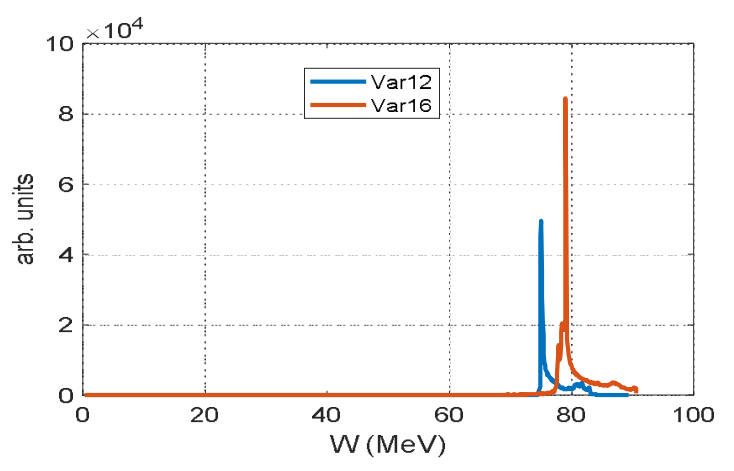

Fig. 8. Energy spectra of the beam at the output of the second section at the maximum energy gain (red curve) and at the minimum phase length of the bunches (blue curve)

As can be seen from the figure, the energy spectrum for the first mode of operation of the first section has a more compact shape, even though the beam energy is slightly lower.

Thus, in our opinion, the mode of operation of the accelerator with a minimum phase length of the bunches at the output of the first section is more suitable in terms of operation of the facility, because it provides both beam energy stability with relatively moderate requirements for microwave phase stability. and changing the energy of the beam in a wide range while maintaining its other parameters, while ensuring more stringent requirements for the stability of the microwave power phase.

\section{SIMULATION OF TRANSPORT LINE}

Based on the obtained results of the self-consistent simulation of particle dynamics in the linac, as well as from the experience of operation of such facilities, we established the following characteristic parameters of the beam at the entrance to the transport line: normalized transverse emittance $\varepsilon_{\mathrm{x}, \mathrm{y}} \mathrm{n} \mathrm{rms}=60 \mathrm{~mm} \cdot \mathrm{mrad}$; beam diameter equals to $3.6 \mathrm{~mm}$; geometric divergence of the beam equals to $0.2 \mathrm{mrad}$. As quadruple lenses, we have chosen wide-aperture lenses, developed at NSC KIPT. Estimates show that their effective length is $315 \mathrm{~mm}$, the field gradient is $35 \mathrm{G} / \mathrm{cm} / \mathrm{A}$, and the winding resistance is $13 \Omega$.

Based on this data, a transportation line model was created for the MAD-X program [12], which is supported free of charge by CERN.

For the convenience of the transport line modeling, the parameters of the quadrupoles were expressed through the excitation currents of their windings and the energy of the beam. We used intrinsic MATCH procedure that automatically adjusts the quadrupole currents for a given energy and the initial transverse parameters of the beam. Restrictive conditions re $21=0$, re $12=0$, re34 $=0$, re $43=0$ provide the transformation of a circular diverging initial beam into a circular beam converging on the target. Fig. 9 shows the result of the transportation line modeling, which confirms this statement. Thus, the transport line can be rearranged for other initial parameters of the beam.

Fig. 10 shows the location of the quadrupoles along the transport line and the beam diameter, calculated from the beta functions for the normalized emittance of $60 \mathrm{~mm} \cdot \mathrm{mrad}$ and the beam energy of $70 \mathrm{MeV}$.

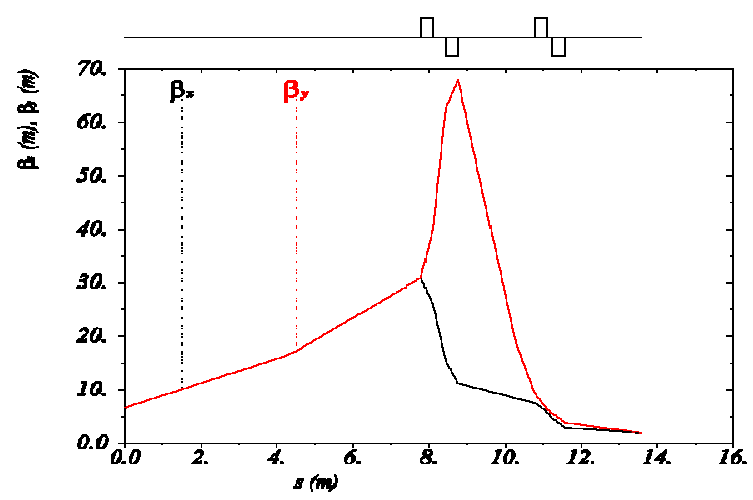

Fig. 9. Beta functions of the beam along the transport line

The convergence of the beam on the target is equal to $0.1 \mathrm{mrad}$ in the horizontal plane and $0.2 \mathrm{mrad}$ in the vertical with a beam diameter of $2 \mathrm{~mm}$.
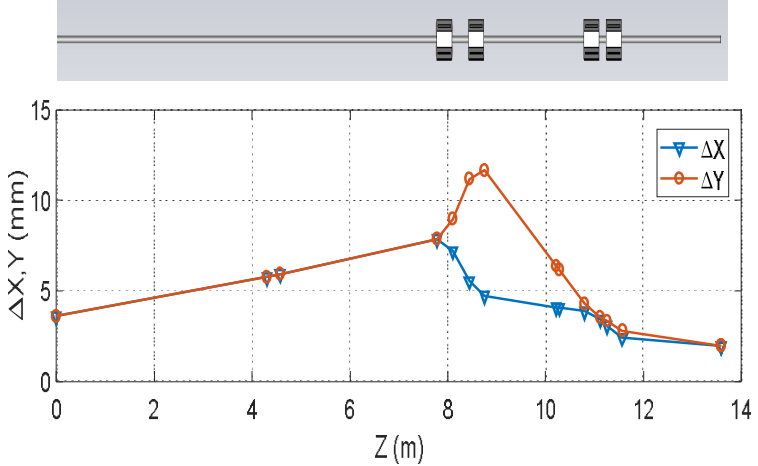

Fig. 10. The position of the quadrupoles and the diameter of the beam along the transport line

The distances from the beginning of the transport line to the center of the quadrupoles and the approximate currents of their excitation are shown in Table 4. It should be noted that at such beam energies, the field gradients of quadrupoles will be proportional to the beam energy to ensure the same beam dynamics. Therefore, for the beam energy of the $80 \mathrm{MeV}$, the currents of the quadrupole windings will be $14 \%$ higher.

At estimated quadrupole winding resistance of $13 \Omega$ their supply voltage will not exceed $30 \mathrm{~V}$, so it is possible to use a list of commercial power supplies with output power of $100 \mathrm{~W}$, for example, SPD3303X to excite the quad-fields. Each of such power supply has two independent power supplies with output voltage up to $32 \mathrm{~V}$ and a current up to $3.2 \mathrm{~A}$. The power supplies have a remote control with a resolution of $1 \mathrm{~mA}, 1 \mathrm{mV}$.

Table 4

Quadrupole parameters

\begin{tabular}{|c|c|c|}
\hline $\begin{array}{c}\text { Quadrupole } \\
\text { number }\end{array}$ & $\begin{array}{c}\text { Distance to } \\
\text { quadrupole, } \mathrm{m}\end{array}$ & $\begin{array}{c}\text { Quadrupole } \\
\text { current, } \mathrm{A}\end{array}$ \\
\hline $\mathrm{Q} 1$ & 8.097 & 1.512 \\
\hline $\mathrm{Q} 2$ & 8.752 & -1.839 \\
\hline $\mathrm{Q} 3$ & 11.111 & 1.230 \\
\hline $\mathrm{Q} 4$ & 11.567 & -0.538 \\
\hline
\end{tabular}

To estimate the diameter of the pipe of the transport line, we proceeded from a size equal to four-fold the maximum dimensions of the beam. Since the maximum beam size is approximately $12 \mathrm{~mm}$, a stainless-steel pipe with an outer diameter of $60 \mathrm{~mm}$ and the wall thickness of $2 \mathrm{~mm}$ is suitable for the beamline. To increase reliabil- 
ity, it is possible to make a transport line of three sections, where the middle section, at the location of the quadrupoles Q1 and Q2, can be made of pipe with a diameter of $100 \mathrm{~mm}$, and the initial and final sections of the pipe of $60 \mathrm{~mm}$. For the convenience of adjusting the regimes of quadrupoles, it is necessary to provide three profilometers: one at the output of the accelerator, the second one at the entrance to the quadrupole lens Q1 and the third one in front of the target as well as at least of two pairs of steering coils along the beamline.

\section{CONCLUSIONS}

An accelerator model has been created for modeling the self-consistent dynamics of electrons taking into account the change in the high-voltage supply of the electron source during the pulse. The simulation at moderate power levels of microwave power supply of the first and the second sections of $12 \mathrm{MW}$ (design power $20 \mathrm{MW}$ ) showed the possibility of obtaining a beam with a pulse current up to $100 \mathrm{~mA}$ in the energy range from 47 to $80 \mathrm{MeV}$. In addition, the results of simulation showed that the accelerator can operate in two main modes: with the maximum energy gain and with the minimum length of the bunches. The regime with a minimum length of bunches is more practical, because it allows to change the energy of the particles in a wide range without significantly changing other parameters, although the maximum energy and beam current are slightly lower in this case. The simulation results correspond to the experimentally measured beam parameters.

The results of simulation have also shown that if the beam at the entrance to the transport line has a normalized emittance of $60 \mathrm{~mm} \cdot \mathrm{mrad}$, diameter $3.6 \mathrm{~mm}$ and energy $70 \mathrm{MeV}$ then we can expect on the target the beam diameter $2 \mathrm{~mm}$ at the beam convergence $0.1 \mathrm{mrad}$ in the horizontal and $0.2 \mathrm{mrad}$ in the vertical planes.

For the convenience of adjusting the quadruple field gradients, it is necessary to provide three profilometers as well as at least two pairs of steering coils along the beamline.

\section{REFERENCES}

1. A. Bezdetko, P. Gladkikh, S. Gokov, et al. The injection system of the NESTOR X-ray generator //
Physics of Particles and Nuclei Letters. 2014, v. 11, p. 615-619.

2. A. Zelinsky, V. Androsov, E. Bulyak, et al. Status of Kharkov X-ray generator based on Compton scattering NESTOR // Proc. of EPAC 04, Lucerne, 2004, p. 2412-2414.

3. M.I. Ayzatskiy, P.G. Gurtovenko, V.F. Zhiglo, et al. Compact electron injector for S-band linac // Problems of Atomic Science and Technology. Series "Nuclear Physics Investigations”. 2008, № 3, p. 68-72.

4. E.Z. Biller, A.N. Dovbnya, V.A. Kushnir, et al. Beam current enhancement in Kharkov electron linac // Particle Accelerators. 1990, v. 27, № 1-4, p. 119-124.

5. Yu.I. Akchurin, V.I. Beloglasov, E.Z. Biller, et al. The accelerator LUE-60 as injector of the technological source of synchrotron radiation // Problems of Atomic Science and Technology. 1989, № 5, p. 3-10.

6. V.V. Mytrochenko, A. Opanasenko. Study of transient self-consistent beam dynamics in rf linacs using the particle tracing code // Nuclear Instruments and Methods in Physics Research A 558, 2006, p. 235-239.

7. L.M. Young. PARMELA. Los Alamos: 1996, 93 p. (preprint / Los Alamos National Laboratory, LAUR-96-1835).

8. J.H. Billen, L.M. Young. POISSON/SUPERFISH on PC compatibles // Proc. 1993 Particle Accelerator Conff. Washington (USA). 1993, p. 790-792.

9. G.A. Loew, R.H. Miller, R.A. Early, K.L. Bane // IEEE Trans. Nucl. Sci. 1979, NS-26, p. 3701.

10. M.I. Ayzatsky, V.F. Zhiglo, V.A. Kushnir, et al. Phase motion of particles upon formation of subpicosecond electron bunches in a traveling wave under heavy beam loading // Physics of Particles and Nuclei Letters. 2010, v. 7, p. 564-567.

11. M.I. Ayzatsky, K.Y. Kramarenko, V.A. Kushnir, et al. Analysis of short electron bunches shaping in a linac // Problems of Atomic Science and Technology. 2018, № 3, p. 81-85.

12. http://mad.web.cern.ch/mad/releases/5.02.08/ madxuguide.pdf

Article received 20.04.2021

\section{ПРОЕКТ ЛИНИИ ТРАНСПОРТИРОВКИ ПУЧКА УСКОРИТЕЛЯ НАКОПИТЕЛЬНОГО КОЛЬЦА НЕСТОР ДЛЯ ОБЕСПЕЧЕНИЯ ЯДЕРНО-ФИЗИЧЕСКИХ ИССЛЕДОВАНИЙ}

\section{В.В. Митроченко, С.А. Пережогин, В.А. Кушнир, В.Б. Ганенко, Н.И. Айзацкий, Г.Д. Коваленко}

Представлены результаты моделирования динамики самосогласованного пучка в линейном ускорителе накопителя НЕСТОР с учетом влияния изменения энергии частиц на выходе электронной пушки во время импульса тока. Полученные данные легли в основу расчета линии транспортировки пучка для исследований в области физики высоких энергий.

\section{ПРОЕКТ ЛІНІЇ ТРАНСПОРТУВАННЯ ПУЧКА ПРИСКОРЮВАЧА НАКОПИЧУВАЛЬНОГО КІЛЬЦЯ НЕСТОР ДЛЯ ЗАБЕЗПЕЧЕННЯ ЯДЕРНО-ФІЗИЧНИХ ДОСЛІДЖЕНЬ}

\section{В.В. Митроченко, С.О. Пережогін, В.А. Кушнір, В.Б. Ганенко, М.І. Айзацький, Г.Д. Коваленко}

Представлено результати моделювання самоузгоденої динаміки пучка в прискорювачі накопичувального кільця НЕСТОР з урахуванням впливу зміни енергії частинок на виході електронної гармати під час імпульсу струму. Отримані дані лягли в основу розрахунку лінії транспортування пучка для забезпечення досліджень у галузі фізики високих енергій. 